\title{
Evaluación online orientada al aprendizaje universitario: Impacto del feedback en los resultados de los estudiantes
}

\author{
Fermín NAVARIDAS-NALDA \\ Ana GONZÁLEZ-MARCOS \\ Fernando ALBA-ELÍAS
}

Datos de contacto:

Fermín Navaridas-Nalda Universidad de La Rioja fermin.navaridas@unirioja.es

Ana González-Marcos Universidad de La Rioja ana.gonzalez@unirioja.es

Fernando Alba-Elías Universidad de La Rioja fernando.alba@unirioja.es

\begin{abstract}
RESUMEN
Investigaciones recientes interesadas en garantizar la calidad de la Educación Superior subrayan con fuerza la conveniencia de introducir cambios en el modo tradicional de orientar la evaluación durante la actividad didáctica. Convencidos de su importancia, el presente estudio tiene como objetivo principal analizar el impacto del feedback en un sistema de evaluación online diseñado desde un enfoque formativo para promover y mejorar el aprendizaje de estudiantes universitarios. La intervención se realizó durante dos cursos académicos consecutivos con 90 estudiantes de ingeniería pertenecientes a titulaciones de grado y máster de la Escuela Técnica Superior de Ingeniería Industrial de la Universidad de La Rioja. Los análisis efectuados indican el efecto positivo que tiene el sistema propuesto, tanto en el rendimiento de los estudiantes como en su grado de satisfacción con la evaluación y feedback proporcionados. Entre las conclusiones obtenidas, cabe destacar que el sistema de evaluación diseñado permite proporcionar información que ayuda a los estudiantes a identificar a tiempo sus puntos débiles en el proceso de aprendizaje y a mejorar su propia cualificación.
\end{abstract}

PALABRAS CLAVE: Evaluación; Retroalimentación; Educación Superior; Ingeniería. 


\title{
Online assessment for learning in higher education: Impact of feedback on students' outcomes
}

\begin{abstract}
Recent research, which is interested in ensuring the quality of Higher Education, strongly emphasizes the necessity of introducing changes in the traditional way of performing assessment during the didactic activity. Because we are convinced about its importance, this study aims to analyze the impact of feedback on an online evaluation system that is designed from a formative perspective to promote and improve the learning process of university students. The intervention was carried out during two consecutive academic courses with 90 undergraduate and master engineering students from the Higher Technical School of Industrial Engineering of the University of La Rioja. The performed analyses point to a positive effect of the proposed online system, both in student performance and in their degree of satisfaction with the assessment and feedback provided. It could be concluded that the designed assessment and feedback system provides information that helps students to identify their weaknesses and to improve their own qualification.
\end{abstract}

KEYWORDS: Assessment; Feedback; Higher Education; Engineering

\section{Introducción}

La actividad docente en cualquier etapa del sistema educativo debería estar guiada por una continua aspiración de mejorar la calidad del aprendizaje, posibilitando a los estudiantes información relevante sobre la que puedan tomar decisiones adecuadas para abordar con éxito la construcción de su propio conocimiento (Santín \& Sicilia, 2015; Dawson et al., 2019; Ibarra-Sáiz \& Rodríguez-Gómez, 2020). En el caso concreto de la Educación Superior, esta concepción constructivista del aprendizaje ha dado lugar a cambios sustanciales en la forma de enfocar y diseñar la práctica educativa. Ahora, por ejemplo, los estudiantes ocupan un papel más central y activo en el diseño de todos los procesos de aprendizaje, asumiendo así una responsabilidad importante durante su desarrollo que antes quizás no tenían. Para algunos autores (Zimmerman, 2001; Pintrich \& Zusho, 2002), asumir dicha responsabilidad permite a los estudiantes establecer metas valiosas para su propio aprendizaje, así como supervisar, controlar y regular su 
pensamiento, motivación y comportamiento durante la actividad de estudio, todo ello en relación con las características, condiciones o exigencias curriculares que plantea el contexto de enseñanza.

Sobre el contexto de enseñanza universitaria, hay numerosas investigaciones (Diez et al., 2009, Biggs \& Tang, 2011, Baeten, Dochy, Struyven, Parmentier, \& Vanderbruggen, 2016) que ponen de relieve la influencia positiva de sus componentes curriculares (tales como los objetivos, la metodología o el sistema de evaluación) en la optimización de los procesos de trabajo y la calidad de los resultados de aprendizaje de los estudiantes. Sin embargo, dentro del nuevo marco de actuación del Espacio Europeo de Educación Superior, sorprende en cierta medida que el cambio e innovación didáctica sobre estos componentes haya sido significativamente distinto tanto en el punto de partida como en el tiempo en producirse en la práctica docente (Nicol y Macfarlane-Dick, 2007). Así, y a pesar del enorme interés que durante los últimos años han suscitado los efectos de la evaluación entre el profesorado universitario (Henderson, Ajjawi, Boud, \& Molloy, 2019), las principales líneas de innovación desde este enfoque parecen centrarse con mayor frecuencia en el ámbito metodológico y en el de los recursos tecnológicos (Pérez, 2018). Con el fin de resolver este relativo desajuste didáctico y dar una respuesta más eficaz a los nuevos desafios docentes que plantea la Educación Superior, estudios recientes instan al profesorado a investigar en/desde la propia acción evaluadora (Henderson, Ryan y Phillips, 2019; Panadero, Broadbent, Boud, \& Lodge, 2019).

Esta situación constituye en gran medida el problema objeto de estudio en nuestro trabajo, llevándonos a replantear el sistema de evaluación como una estrategia docente para mejorar la calidad del aprendizaje de los estudiantes universitarios, reconsiderando al mismo tiempo su punto de partida en el proceso de planificación. En este sentido, nos preguntamos algunas cuestiones como las que siguen: ¿qué elementos o características definen el carácter formativo de un sistema de evaluación? De acuerdo con el marco normativo que regula la evaluación convencional en nuestra universidad, y atendiendo al nuevo paradigma de la Educación Superior contemporánea, ¿es posible diseñar un sistema de evaluación sostenible que resulte compatible con la doble finalidad que se plantea en el aprendizaje universitario: a) por una parte, calificar o certificar el nivel de logro alcanzado por los estudiantes al final de un curso o programa (finalidad sumativa), y b) por otra, apoyar de manera continua los procesos de trabajo de los estudiantes para que puedan satisfacer sus necesidades formativas presentes y lo transformen en acciones de mejora para un futuro próximo o trabajo posterior (finalidad formativa)?

Con relación a estas cuestiones, encontramos abundante literatura 
sobre evaluación formativa (también conocida como evaluación para el aprendizaje) donde se destaca de forma unánime la información de retorno (feedback) como el elemento formal más importante para definir su carácter (Sadler, 1989; Black \& Willian, 1998; Hattie \& Timperley, 2007; Nicol \& Macfarlane-Dick, 2007; Morales, 2010; Bennett, 2011; Carless \& Boud, 2018). Sobre la base de estos mismos trabajos, podemos llegar concebir la evaluación formativa como un proceso (o una serie organizada de procesos) generador de información relevante sobre la actuación del estudiante orientado a facilitar la construcción personal de su aprendizaje y la mejora posterior de su rendimiento. En esta misma línea de pensamiento, Henderson et al. (2019) subrayan la importancia del impacto o los efectos que ocurren en el proceso de feedback como una característica básica y necesaria para responder con eficacia a los propósitos de la evaluación formativa. De esta forma, dichos autores defienden que cualquier información sin impacto (es decir, sin cambios o mejoras significativas en la condición del estudiante) no es feedback, tan solo transmisión de información que carece de valor o relevancia para la formación del estudiante.

De acuerdo con esta idea, algunos autores (Boud \& Molloy, 2013; Ryan, Gašević, \& Henderson, 2019) parecen constatar que el feedback proporcionado a los estudiantes universitarios a menudo se limita a comentarios o resultados académicos en su forma acabada después del proceso formativo (por ejemplo, calificaciones obtenidas, número de respuestas correctas, errores cometidos, fortalezas o debilidades identificadas). Sensibles con la necesidad de cambio de esta realidad educativa, algunas investigaciones recientes en el ámbito universitario (Delva et al., 2013; Winstone, Rowntree, \& Parker, 2017; Dawson et al., 2019) ponen de manifiesto que la eficacia de la evaluación formativa depende en buena medida del grado de participación de los estudiantes en el proceso de retroalimentación, así como de su actuación de acuerdo con la información de retorno que generan, solicitan o reciben en dicho proceso (estudiantes proactivos). Desde esta perspectiva, cabe presumir que la calidad del aprendizaje no mejora de forma automática con un proceso unidireccional de feedback por parte del profesorado. Más aún, como aciertan a señalar Nicol y Macfarlane-Dick (2007), si la responsabilidad de la evaluación recae de forma exclusiva en manos de profesor (estudiantes reactivos), entonces será dificil que los estudiantes puedan adquirir y desarrollar competencias que en la actualidad se consideran claves para su desarrollo personal y profesional futuro (tanto en lo que se refiere a su dimensión cognitiva, como en lo relativo a otras dimensiones de su vida afectiva-emocional muy relacionadas con la capacidad de autorregulación, control y juicio crítico del propio desempeño competencial). 
Este cambio tan significativo en la forma de comprender el feedback en la actividad evaluadora, ha despertado el interés de numerosos investigadores por identificar los aspectos que pueden contribuir a mejorar su impacto en el aprendizaje (Hattie, 2008; Winstone et al., 2017; Pardo, Jovanović, Dawson, Gašević, \& Mirriahi, 2019; Ryan, Gašević, \& Henderson, 2019). En general, los aspectos más destacados se refieren a la relevancia de la información facilitada (orientada a que los estudiantes puedan satisfacer sus propias necesidades futuras de aprendizaje, comentarios útiles para abordar nuevas tareas), la pertinencia (información personalizada, adaptada a las características y necesidades individuales de aprendizaje), la eficiencia (comentarios realizados a tiempo, oportunos y rápidos para implementar acciones de mejora) y la claridad (comentarios precisos para asegurar la comprensión de la información facilitada o generada, evitando en lo posible incertidumbres, ambigüedad o dudas de interpretación). De esta manera, el profesor toma un papel muy importante en el diseño de los procesos de feedback (Carless, 2015; Henderson et al., 2019), facilitando a los estudiantes oportunidades para desarrollar su juicio evaluativo sobre la calidad del trabajo realizado, así como la reflexión crítica sobre lo que han aprendido y lo que pueden utilizar en un desempeño posterior (Tai, Aijawi, Boud, Dawson \& Panadero, 2018). En este sentido, y con el foco de atención en la mejora del impacto del feedback, parece recomendable que estas decisiones docentes encaminadas a promover el juicio evaluativo contemplen una variedad significativa de fuentes, agentes educativos y tipos de información (Boud \& Molloy, 2013; Panadero et al., 2019).

Así, con todo, las actuales circunstancias que está viviendo la universidad del aprendizaje (por ejemplo, en lo relativo a los nuevos modelos organizativos de aula, las ratios de estudiantes por profesor, los sistemas de agrupamiento del alumnado, o la asignación de tiempos de trabajo presencial y fuera del aula) plantean un verdadero desafio al profesorado con respecto al diseño de la evaluación en su doble finalidad: sumativa y formativa. Para algunos autores (Bennett, 2011; Carless, 2015), la evaluación formativa bien pensada puede (debe) integrar de forma armónica el propósito primario de la evaluación tradicional sin necesidad de añadir una mayor carga de trabajo docente al profesorado universitario. En este sentido, la investigación en este campo (Donia, O'Neill, \& Brutus, 2018; Pardo et al., 2019; Winstone, 2019) destaca el papel cada vez más importante que desempeña la tecnología, proporcionando soluciones muy innovadoras en tareas complejas como son el seguimiento, el control y el análisis del aprendizaje, además de optimizar los procesos de feedback y de reducir de forma significativa la carga de trabajo del profesor. 
En consecuencia, nos proponemos diseñar un modelo de evaluación online con un marcado enfoque formativo para mejorar la adquisición de competencias en estudiantes de ingeniería, de grado y máster. Dicha finalidad se concreta, en este trabajo, en analizar el impacto que tiene el sistema de evaluación y feedback propuesto en el rendimiento de los estudiantes a lo largo de su proceso formativo. Así mismo, se analiza el nivel de satisfacción de los estudiantes con el modelo propuesto.

\section{Método}

\section{Muestra}

En el estudio, llevado a cabo durante dos cursos académicos consecutivos, participaron un total de 90 estudiantes de la Escuela Técnica Superior de Ingeniería Industrial (ETSII) de la Universidad de La Rioja que cursaban la materia "proyectos" en el Máster Universitario en Ingeniería Industrial (40 alumnos) y en el Grado en Ingeniería Mecánica (50 alumnos).

\section{Contexto de la investigación}

Con objeto de facilitar el proceso de aprendizaje de los estudiantes, se ha diseñado un modelo mixto de enseñanza que combina clases magistrales con un proceso activo fundamentado en el aprendizaje basado en problemas (PBL), en el que los estudiantes de diferentes titulaciones trabajan en equipo en la realización proyectos de ingeniería del mundo real, proporcionando, de este modo, autenticidad al modelo propuesto (Orgill, 2007).

En todos los casos (tanto en grado como en máster), las clases magistrales tienen una periodicidad semanal y una duración de dos horas. En dichas clases, se desarrolla el contenido específico establecido para cada titulación en la correspondiente materia "proyectos". En concreto, el enfoque de la asignatura de máster está fundamentalmente orientado a la dirección y gestión de proyectos, mientras que la asignatura de grado se centra más en la ingeniería de proyectos.

Respecto a las actividades prácticas, tal como se describe en González-Marcos, Alba-Elías, Navaridas-Nalda y Ordieres-Meré (2016), se utiliza un entorno de simulación con el que es posible recrear la realidad de una organización dedicada a dirigir, gestionar y organizar proyectos. En esencia, los alumnos de grado y máster, que trabajan conjuntamente en equipos de proyecto, reciben el encargo de realizar un proyecto que provea a un cliente (docentes) de un producto funcional, completo, con unas metas y objetivos claramente definidos, y que debe contar con una presentación final en la que se muestren sus 
Evaluación online orientada al aprendizaje universitario: Impacto del feedback en los resultados de los estudiantes

características técnicas y comerciales. Todo ello bajo unas restricciones de coste y plazos, tal como sucede en la realización de proyectos profesionales.

La dirección, gestión y organización de los proyectos (negociación del alcance, gestión de riesgos, programación temporal, etc.), se lleva a cabo siguiendo la metodología de dirección de proyectos PRINCE2 (PRojects IN a Controlled Environment) (Office of Government Commerce, 2009), la cual cuenta con una estructura de proyecto definida en la que existen diferentes roles y responsabilidades. En el caso de la experiencia propuesta, los estudiantes adoptan las figuras más relevantes en un proyecto, a saber:

- Ejecutivo/a (EX). Formando parte de la denominada "junta de proyecto", este rol es el encargado de la gestión efectiva del proyecto, siendo el máximo responsable de entregar al cliente el proyecto solicitado. Dos o tres alumnos de máster adoptan este rol durante toda la ejecución del proyecto.

- Director/a de Proyecto (PM). En nombre de la junta de proyecto $(\mathrm{EX})$, los directores de proyecto tienen la autoridad para ejecutar el día a día del proyecto, siendo responsables de que se generen los productos necesarios para alcanzar la calidad requerida, dentro del tiempo y los costes establecidos. Entre seis y nueve estudiantes de máster ejercen las funciones propias de este rol.

- Director/a de equipo (TMg). Esta figura es la encargada de gestionar equipos de ingenieros de proyecto. Así, partiendo de la solicitud realizada por el equipo de directores de proyecto (PM), organiza, dirige y controla el trabajo del equipo de ingenieros de proyecto para producir y entregar los productos solicitados. En el transcurso del desarrollo del proyecto, y de forma temporal, algunos estudiantes de máster cambian su rol de PM a TMg.

- Ingeniero/a de proyecto (TM). Este rol no tiene responsabilidad de gestión, sino que su principal actividad se centra en el desarrollo de las tareas de ingeniería necesarias para generar los productos solicitados. Cada equipo de ingenieros de proyecto está constituido por entre 13 y 15 alumnos de grado.

De este modo, y en consonancia con los objetivos de cada asignatura, los alumnos de máster pueden desarrollar las competencias más relacionadas con la dirección y gestión de proyectos, y los alumnos de grado pueden poner en práctica competencias más propias de la ingenieria de proyectos. En la Figura 1, se muestra una representación gráfica de los roles desempeñados. 
Por su parte, el equipo docente también adopta distintos roles, más allá de la función tradicional de transmisor y evaluador de conocimientos, esto es, propiedad (o Corporate, según la terminología propia de PRINCE2), auditor, consultor experto en organización y documentación, etc. (ver Figura 1).

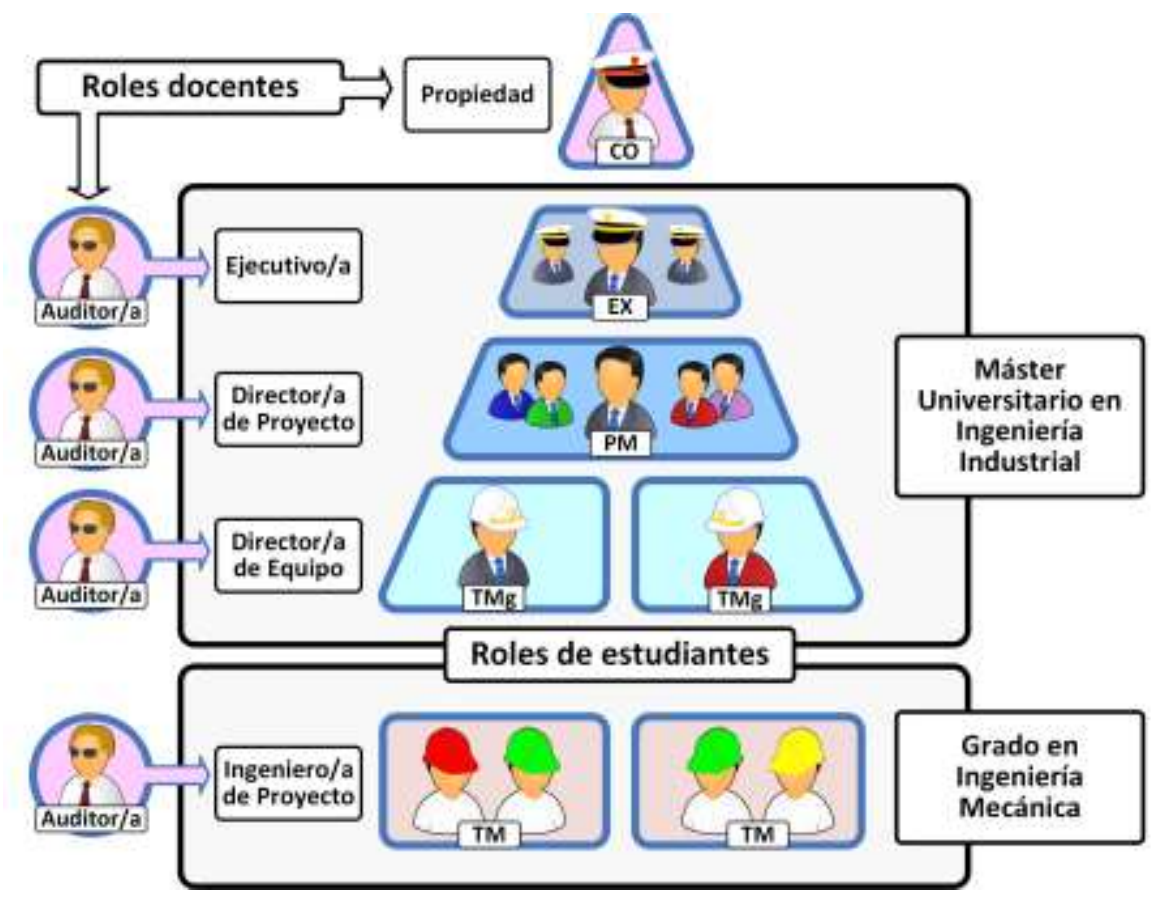

Figura 1. Roles de los distintos participantes en cada proyecto: docentes (Propiedad - CO -, auditor), alumnos de máster (EX, PM, TMg) y grado (TM).

\section{Instrumentos}

El marco de referencia de las competencias a poner en práctica y desarrollar durante la ejecución de los proyectos ha sido el ICB (IPMA Competence Baseline) de IPMA (International Project Management Association) (IPMA, 2006). Para la evaluación regular del grado de adquisición de dichas competencias por parte de cada estudiante, tal como se describe en González-Marcos, Alba-Elias y Ordieres-Meré (2016), se ha diseñado un conjunto de cuestionarios en los que los participantes (docentes y alumnos) deben responder a unas preguntas sobre la calidad de los productos generados, de la ejecución de los procesos de PRINCE2, así como sobre determinados aspectos relacionados con algunas competencias de comportamiento (trabajo en equipo, liderazgo, compromiso y motivación, y orientación a resultados). 
Esta evaluación contempla la valoración por parte de cada estudiante de diferentes escenarios, así como la elaboración de análisis reflexivos sobre los mismos. Además, tanto los docentes como otros participantes del proceso formativo, evalúan las mismas acciones y escenarios. Todo ello con el objetivo de tener una visión lo más amplia posible de cada escenario: la del propio estudiante, la de sus pares y la de los docentes.

Los resultados de estas evaluaciones se entregan de manera periódica a los alumnos a través de la plataforma web de integración que ha sido creada específicamente para el desarrollo de esta experiencia. Así, cada estudiante recibe la siguiente información individual de detalle:

- Contribución de cada producto del proyecto desarrollado o acción realizada en su calificación actual.

- Nivel de desempeño de las competencias evaluadas y su contribución a la calificación actual.

Esa información de carácter individual se complementa con informes detallados sobre las buenas y malas prácticas identificadas en ese periodo del proyecto (lecciones aprendidas). En sesiones específicas, se analiza esta información de forma colectiva para identificar qué ha funcionado, qué no ha funcionado en el proyecto y cómo se debería proceder en el futuro (proalimentación).

Por otro lado, los estudiantes registran su opinión acerca del sistema de evaluación y feedback empleado durante el desarrollo del proyecto en un cuestionario diseñado específicamente para tal fin. Concretamente, el grado de satisfacción mostrado por los estudiantes se recoge mediante las siguientes cuestiones:

Q1: El sistema de evaluación del proyecto me ha motivado a realizar las actividades del mismo.

Q2: El sistema de evaluación del proyecto aporta información útil para saber qué hacer en cada momento de la ejecución del proyecto.

Q3: Mi calificación mejorará en los siguientes feedback.

Q4: La representación gráfica del esfuerzo, calificación y rendimiento me parece útil.

Q5: Creo que es positivo que me faciliten el feedback en distintos momentos.

Q6: De manera general, el sistema de evaluación + feedback, me ha ayudado a saber qué hacer en cada fase del proyecto.

Cada estudiante responde a estos ítems atendiendo a una escala de 1 a 5 de tipo Likert (donde 1 indica Nada, nunca, y 5 indica Mucho, siempre).

\section{Procedimiento}

A lo largo del desarrollo de cada proyecto, y conforme al sistema diseñado para la evaluación de competencias, cada estudiante evalúa 
su propio trabajo, así como el del resto de los participantes en el proyecto con los que interacciona. Así mismo, el equipo docente evalúa las actividades desarrolladas por cada estudiante. Nótese que los formularios empleados por todos los participantes (estudiantes y docentes) son los mismos, ya que el objetivo es recoger información continua acerca de las mismas acciones y escenarios, pero desde diferentes puntos de vista.

Con todas estas opiniones basadas en evidencias, asi como con las evidencias analíticas que es posible extraer de los sistemas de información que dan soporte al entorno de simulación empleado, es posible conocer la evolución de las competencias adquiridas por cada estudiante y proporcionar retroalimentación que permita identificar puntos débiles y ayude a mejorar su propia cualificación.

Tal como se ha indicado previamente, cada estudiante recibe, como mínimo, tres tipos distintos de feedback. Dicha información se proporciona en tres momentos del desarrollo del proyecto, los cuales coinciden con el fin de cada fase del proyecto.

Existe, además, una herramienta a disposición de los estudiantes, que les permite solicitar, en cualquier momento del desarrollo del proyecto, una auditoría sobre la integridad de algunas de las acciones $\mathrm{y}$ procedimientos llevados a cabo en el proyecto, y que están relacionados con la planificación del trabajo, asignación de recursos, etc.

Después de cada evaluación y feedback emitido, los estudiantes deben cumplimentar el cuestionario específico sobre su grado de satisfacción con el sistema empleado.

\section{Análisis estadísticos}

Tras un análisis descriptivo, y con el fin de investigar si se observan diferencias significativas en la evolución de los estudiantes, se emplea el test no paramétrico de los rangos con signo de Wilcoxon, ya que las condiciones de normalidad de la muestra no se cumplen. El nivel de significancia establecido es 0.05 .

\section{Resultados}

En primer lugar, se analiza la eficacia del modelo de evaluación y feedback propuesto, a través de la evolución del rendimiento obtenido por los estudiantes. De acuerdo con De la Fuente et al. (2008), este rendimiento se define como el grado o nivel de cumplimiento de los 
estándares de aprendizaje especificados en el proyecto con relación a lo que los estudiantes deben conocer, comprender y saber hacer desde un enfoque de competencias y en un periodo de tiempo adecuado. Así, en la Figura 2, se presenta, por titulación, el rendimiento medio (en porcentaje) que obtuvieron los estudiantes a la finalización de cada una de las tres fases en las que se desarrolló cada proyecto. Además, en dicha figura se representa el intervalo de confianza (IC) obtenido con una probabilidad del $95 \%$. Cabe señalar que, según la metodología PRINCE2, el arranque del proyecto está enfocado exclusivamente en su organización (definición del alcance, planificación, etc.). De ahí, que los estudiantes de grado no realicen actividades durante la primera fase, la cual suele durar, aproximadamente, dos semanas.

Aunque se observa una evolución positiva en el rendimiento de los estudiantes a lo largo de todo el proyecto, el test no paramétrico de los rangos con signo de Wilcoxon llevado a cabo, no identifica diferencias estadísticamente significativas entre los rendimientos obtenidos en cada fase. En cualquier caso, como sugieren los resultados obtenidos en trabajos similares (Ajjawi \& Boud, 2018; Pardo et al., 2019), el mayor incremento en el nivel de desempeño de los estudiantes se observa tras la primera retroalimentación realizada, tanto en grado como en máster. Del mismo modo, Gibbs y Simpson (2004) llegan a concluir que para asegurar un impacto positivo en los resultados de aprendizaje es necesario que los estudiantes obtengan o generen feedback a tiempo, de modo que puedan implementar acciones de mejora a lo largo de su proceso de aprendizaje.

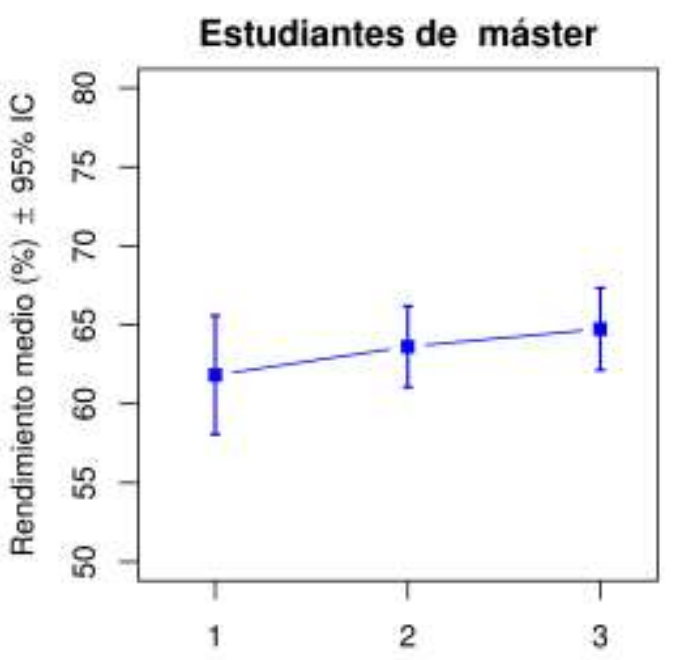

Fase del proyecto

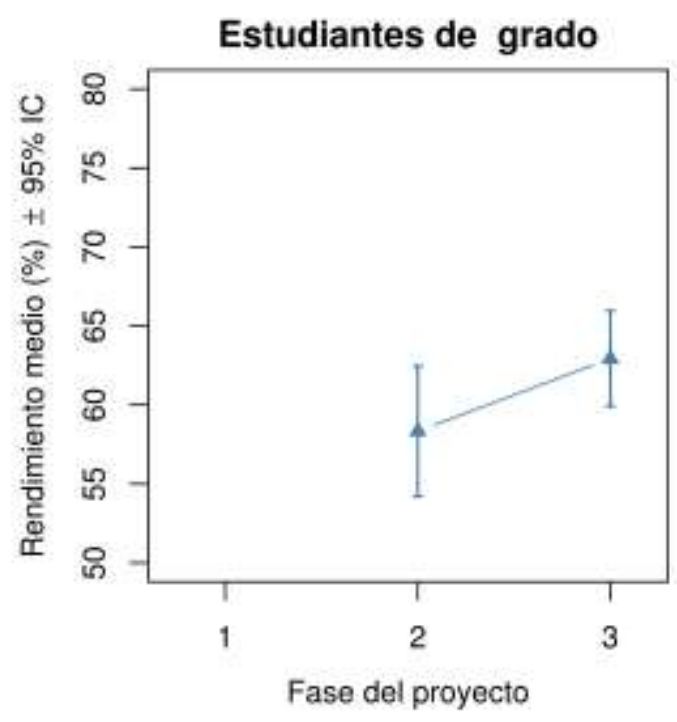

Fase del proyecto

Figura 2. Evolución del rendimiento de los estudiantes a lo largo del 
proyecto en función de su titulación: máster (izquierda) y grado (derecha).

Por otro lado, tal como se ilustra en la Figura 3, los alumnos muestran un elevado nivel de satisfacción con el sistema de evaluación y feedback empleado, considerándolo motivador (Q1) y útil (Q2 y Q4). De hecho, sus expectativas de mejora en las siguientes fases del proyecto (Q3) también son altas. Para algunos autores (Pintrich \& Zusho, 2002; Nicol \& Macfarlane-Drick, 2007), estas son condiciones necesarias para mejorar la calidad de los procesos de aprendizaje de los estudiantes (por ejemplo, en lo relativo a su autorregulación y control cognitvo, actitudinal o conductual en las tareas académicas), así como para conseguir un mayor nivel de logro en los resultados de aprendizaje esperados tanto a nivel individual como grupal (Ibarra-Sáiz \& Rodríguez-Gómez, 2020).

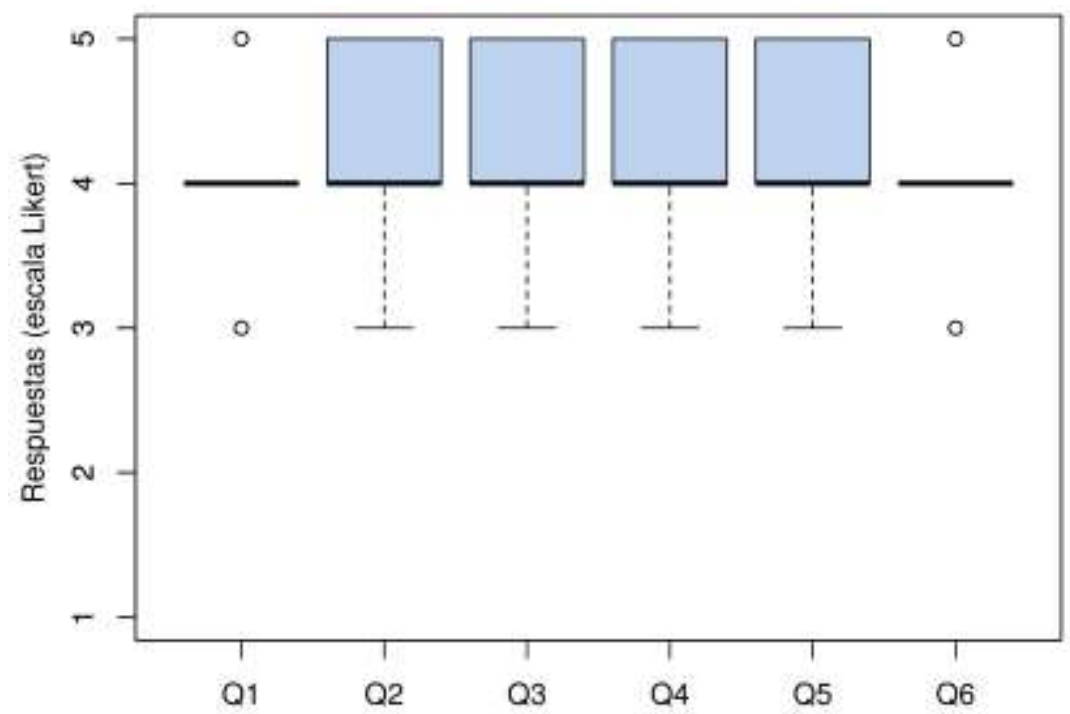

Figura 3. Distribución de las respuestas de los estudiantes sobre su grado de satisfacción con el modelo de evaluación y feedback empleado.

Por último, cabe destacar que los alumnos valoran positivamente el hecho de recibir feedback en distintos momentos del proceso formativo (Q5), además de considerar que el sistema presentado en este trabajo les ha permitido identificar cómo deberian proceder en el futuro (Q6), lo que parece reforzar la idea de que la metodología propuesta no solo permite calificar el nivel de logro alcanzado (evaluación sumativa), sino que también favorece que los estudiantes transformen lo aprendido en acciones de mejora en trabajos posteriores dentro del mismo proyecto (evaluación formativa). Estos resultados vienen a confirmar en gran 
medida las conclusiones obtenidas por otros autores en trabajos similares dentro del mismo ámbito de la ingeniería y sobre la misma base de un modelo mixto de enseñanza mediada con recursos tecnológicos (Pardo, 2018; Pardo et al., 2019).

\section{Conclusiones}

En este trabajo se presenta un sistema de evaluación de competencias y feedback, aplicado a la materia "proyectos" que cursan estudiantes de ingeniería, tanto en grado como en máster, de la ETSII de la Universidad de La Rioja. Con el fin de garantizar la eficacia del feedback, y al igual que en otros estudios similares (Johnson \& Johnson, 1993; Rodríguez-Gómez, Ibarra-Sáiz, \& Gómez, 2011; Boud \& Molloy, 2013; Dawson et al., 2019; Panadero et al., 2019), la evaluación de competencias se realiza a través de un conjunto de indicadores de realización o desempeño (opiniones basadas en evidencias y evidencias analíticas) de las distintas acciones llevadas a cabo por los estudiantes durante el desarrollo de un proyecto, contemplando en su diseño una variedad significativa de mecanismos, prácticas, fuentes y tipos de información para facilitar en todo lo posible el compromiso y la participación efectiva de los estudiantes en su propia evaluación (estudiantes proactivos).

Dado que la evaluación continua de los estudiantes se apoya en buena medida en las respuestas emitidas, a través de un conjunto de cuestionarios online, por todos los agentes que participan en el proceso educativo (estudiantes, equipo docente), el sistema de evaluación propuesto sería transferible a otras materias con una adaptación de las preguntas realizadas al contexto específico de cada materia. Además, cabe destacar la relevancia y el valor didáctico del sistema online propuesto en situaciones formativas de no presencialidad. Esta conclusión toma especial importancia con la investigación realizada por Rodríguez y Salinas (2020), donde se advierte una demanda urgente por parte del profesorado universitario de modelos, procedimientos e instrumentos que permitan adaptar la actividad evaluadora a contextos de aprendizaje activo en los que se utilicen medios electrónicos (semipresencial/blended-learning, no- presencial/e-learning).

De este modo, comprobamos que es posible obtener una visión integrada, válida y fiable de las competencias adquiridas por cada estudiante durante los procesos de aprendizaje, en lugar de estar sujetos a un único valor o dato proporcionado por un sistema de evaluación sumativa para el conjunto del proceso formativo. Estos resultados coinciden en buena medida con los obtenidos en un modelo de evaluación de características similares investigado por Carless (2015). 
Además, sobre la base de estos mismos resultados, podemos concluir que el sistema diseñado facilita información relevante que ayuda a los estudiantes a identificar a tiempo sus puntos débiles, controlar y regular su proceso de aprendizaje, así como tomar decisiones personales para mejorar de forma continua su propia cualificación. En este sentido, cabe destacar que todas las características indicadas resultan esenciales para asegurar un impacto positivo en el aprendizaje de los estudiantes (Winstone et al., 2017; Ryan et al., 2019).

De acuerdo con todo ello, es posible valorar positivamente el impacto que tiene el sistema de evaluación y feedback propuesto en el rendimiento académico de los estudiantes. Así mismo, podemos concluir que los propios estudiantes valoran positivamente el sistema adoptado y consideran que favorece su proceso de aprendizaje.

Como con la mayoría de las investigaciones educativas, este estudio tiene ciertas limitaciones que se abordarán en futuras investigaciones. Por un lado, sería necesario un tamaño de muestra mayor para sacar conclusiones más generalizables. Por otro, dado que los resultados de este estudio se limitan a dos asignaturas de una misma universidad, investigaciones futuras deberian incorporar otras asignaturas y un conjunto de universidades más amplio. Esto permitiría evaluar tanto el grado de influencia de una materia específica en los resultados, como el efecto en los mismos de los estilos del equipo docente o del propio contexto de la enseñanza (por ejemplo, procedimientos de la institución, recursos, etc.). También convendría profundizar en otras variables de entrada de los propios estudiantes que podrían explicar en parte los resultados obtenidos (por ejemplo, rasgos personales, enfoques de aprendizaje, vía de acceso a la universidad o experiencia laboral). Abordar estas limitaciones mejoraria el valor del presente estudio, ya que permitirán construir un mayor conocimiento para la mejora continua de la actividad docente universitaria.

\section{Agradecimientos}

Los autores desean expresar su agradecimiento al "Vicerrectorado de Profesorado, Planificación e Innovación Docente" de la Universidad de La Rioja, a través de la "Dirección Académica de Formación e Innovación Docente", por el soporte económico otorgado para el desarrollo de este trabajo.

\section{Referencias}

Ajjawi, R. y Boud, D. (2018). Examining the nature and effects of 
Evaluación online orientada al aprendizaje universitario: Impacto del feedback en los resultados de los estudiantes

feedback dialogue. In Assessment \& Evaluation in Higher Education, 43(7), 1106-

1119. DOI: $10.1080 / 02602938.2018 .1434128$

Baeten, M., Dochy, F., Struyven, K., Parmentier, E. \& Vanderbruggen, A. (2016). Student-centred learning environments: an investigation into student teacher's instructional preferences and approaches to learning. Learning Environments Research, 19(1), 43-62. DOI: 10.1007/s10984015-9190-5

Bennett, R. E. (2011). Formative assessment: a critical review. In Assessment in Education: Principles, Policy \& Practice, 18, 5-25. DOI: 10.1080/0969594X.2010.513678

Biggs, J.B. y Tang, C. (2011). Teaching for Quality Learning at University (4th ed.). Berkshire: Open University Press.

Black, P. y Wiliam, D (1998). Inside the Black Box: Raising Standards through Classroom Assessment. London: King's College London School of Education.

Boud, D. y Molloy, E. (2013). Rethinking models of feedback for learning: the challenge of design. In Assessment \& Evaluation in Higher Education, 38(6), 698712. DOI: $10.1080 / 02602938.2012 .691462$

Carless, D. (2015). Exploring learning-oriented assessment processes. In Higher Education, 69, 963-976. DOI: 10.1007/s10734-014-9816-Z

Carless, D. y Boud, D. (2018). The development of student feedback literacy: enabling uptake of feedback. In Assessment \& Evaluation in Higher Education, 43(8), 13151325. DOI: $10.1080 / 02602938.2018 .1463354$

Dawson, Ph., Henderson, M., Mahoney, P., Phillips, M., Ryan, T., Boud, D. y Molloy, E. (2019). What makes for effective feedback: staff and student perspectives. In Assessment \& Evaluation in Higher Education, 44(1), 2536. DOI: $10.1080 / 02602938.2018 .1467877$

De la Fuente, J., Pichardo, M.C., Justicia, F. y Berbén, A. (2008). Enfoques de aprendizaje, autorregulación y rendimiento en tres universidades europeas. En Psicothema, 20(4), 705-711. 
Recuperado

https: / / www.redalyc.org/articulo.oa?id=727/72720430

Delva, D., Sargeant, J., Miller, S., Holland, J., Brown, P., Leblanc, C., Lightfoot, K. y Mann, K. (2013). Encouraging residents to seek feedback. In Medical Teacher, 35(12), pp. e1625e1631. DOI: 10.3109/0142159X.2013.806791

Diez, M.C., Pacheco, D.I., García, J.N., Martínez, B., Robledo, P., Álvarez, M. L., Carbonero, M. A., Román, J.M., Del Caño, M. y Monjas, I. (2009). Percepción de los estudiantes universitarios de educación respecto al uso de metodologías docentes y el desarrollo de competencias ante la adaptación al EEES: datos de la Universidad de Valladolid. Aula Abierta, 37(1), 45-56.

Donia, M. B., O'Neill, T. A., y Brutus, S. (2018). The longitudinal effects of peer feedback in the development and transfer of student teamwork skills. In Learning and Individual Differences, 61, 87-98. DOI: 10.1016/j.lindif.2017.11.012.

Gibbs, G., y Simpson, C. (2004). Conditions under which assessment supports students' learning. In Learning and Teaching in Higher Education, 1(1), 3-31. Recuperado de http://eprints.glos.ac.uk/id/eprint/3609

González-Marcos, A., Alba-Elías, F., Navaridas-Nalda, F., y OrdieresMeré, J. (2016). Student evaluation of a virtual experience for project management learning: An empirical study for learning improvement. Computers \& Education, 102, 172-187. DOI: $10.1016 /$ j.compedu.2016.08.005

González-Marcos, A., Alba-Elías, F., y Ordieres-Meré, J. (2016). An analytical method for measuring competence in project management. British Journal of Educational Technology, 47(6), 1324-1339. DOI: 10.1111 /bjet.12364

Hattie, J. (2008). Visible learning: a synthesis of over 800 metaanalyses related to achievement. New York: Routledge. DOI: $10.4324 / 9780203887332$

Hattie, J. y Timperley, H. (2007). The power of feedback. In Review of Educational Research, 77(1), 81-112. DOI: 10.3102/003465430298487

Henderson, M., Ajjawi, R., Boud, D. y Molloy, E. (Eds.). (2019). The 
Evaluación online orientada al aprendizaje universitario: Impacto del feedback en los resultados de los estudiantes

Impact of Feedback in Higher Education: Improving Assessment

Outcomes for Learners. Cham Switzerland: Palgrave Macmillan. Recuperado de https://doi.org/10.1007/978-3030-25112-3

Henderson, M., Ryan, T. y Phillips, M. (2019). The challenges of feedback in higher education, Assessment \& Evaluatio. In Higher Education, 44(8), 1237-1252. DOI: 10.1080/02602938.2019.1599815

Ibarra-Sáiz, M. S. y Rodríguez-Gómez, G. (2020). Aprendiendo a evaluar para aprender en la educación superior. Revista Iberoamericana de Evaluación Educativa, 13(1), 5-8. Recuperado de https://doi.org/10.15366/riee2020.13.1

IPMA (2006). ICB: IPMA Competence Baseline. Version 3.0. Nijkerk, The Netherlands: International Project Management Association.

Johnson, D., y Johnson, R. (1993). Cooperative learning and feedback in technology-based instruction. In Dempsey, J. y Sales, G. (Eds.). Interactive instruction and feedback (pp. 133157). Englewood Cliffs, NJ: Educational Technology Publications.

Morales, P. (2010). La evaluación formativa. En Morales, P. Ser profesor: una mirada al alumno, $2^{a}$ edición (capítulo II, pp. 3390). Guatemala: Universidad Rafael Landivar, Recuperado de http://www.upcomillas.es/personal/peter/otrosdocumentos / Evaluacionformativa.pdf

Nicol, D. J. y Macfarlane-Drick, D. (2007). Formative assessment and self-regulated learning: a model and seven principles of good feedback practice. Studies in Higher Education, 31, 199218. DOI: $10.1080 / 03075070600572090$

Office of Government Commerce (2009). Managing Successful Projects with PRINCE2 ${ }^{T M}$. Norwich, UK: The Stationary Office.

Orgill, M. (2007). Situated cognition. In Bodner, G. M. y Orgill, M. (Eds.). Theoretical frameworks for research in chemistry/science education (pp. 187-203). Upper Saddle River, NJ: Prentice Hall.

Panadero, E., Broadbent, J., Boud, D. y Lodge, J. M. (2019). Using 
formative assessment to influence self- and co-regulated learning: the role of evaluative judgement. In European Journal of Psychology of Education, 34(3), 535-557. DOI: 10.1007/s10212-018-0407-8

Pardo, A. (2018). A feedback model for data-rich learning experiences. In Assessment \& Evaluation in Higher Education, 43(3), 428-438. DOI: https://doi.org/10.1080/02602938.2017.1356905.

Pardo, A., Jovanović, J., Dawson, S., Gašević, D., y Mirriahi, N. (2019). Using learning analytics to scale the provision of personalised feedback. In British Journal of Educational Technology, 5O(1), 128-138. DOI: 10.1111 /bjet.12592.

Pérez, J. (2018). La calidad y la evaluación en la Educación Superior. En Carrasco, S. y Corral, I. (coords). Docencia universitaria e innovación. Evolución y retos a través de los CIDUI (pp. 137152). Barcelona: Octaedro. Recuperado de https: / /www.cidui.org/wp-content/uploads/2018/07/ePDFDocenciaUniversitariaEInnovacion.pdf

Pintrich, P.R. y Zusho, A. (2002). Student motivation and selfregulated learning in the college classroom. In Higher Education: handbook of theory and research (Vol. XVII, pp. 731810). New York: Agathon Press.

Rodríguez-Gómez, G., Ibarra-Sáiz, M. S. y Gómez, M. A., (2011). eAutoevaluación en la universidad: un reto para profesores y estudiantes. Revista de Educación, 356, 401-430. DOI: 104438/1988-592X-RE-2010-356-045

Rodríguez, H.M. y Salinas, M.L. (2020). La Evaluación para el Aprendizaje en la Educación Superior: Retos de la Alfabetización del Profesorado. Revista Iberoamericana de Evaluación Educativa, 13(1), 111-137. Recuperado en https: / /doi.org/10.15366/riee2020.13.1.005

Ryan, T., Gašević D. y Henderson, M. (2019). Identifying the Impact of Feedback Over Time and at Scale: Opportunities for Learning Analytics. In Henderson M., Ajjawi R., Boud D., Molloy E. (Eds). The Impact of Feedback in Higher Education (pp. 207-223) Palgrave Macmillan, Cham. DOI: 10.1007/978-3-030-251123_12 
Evaluación online orientada al aprendizaje universitario: Impacto del feedback en los resultados de los estudiantes

Sadler, D. R. (1989). Formative assessment and the design of instructional systems. In Instructional Science, 18(2), pp. 119144. DOI: $10.1007 / \mathrm{BF} 00117714$

Santín, D. y Sicilia, G. (2015). Evaluar para mejorar: hacia el seguimiento y la evaluación sistemática de las políticas educativas. En Santín, D., Balart, P., Cabrales, A., Calero, J., De la Fuente, A., Escardíbul, J. O., Felgueroso, F. y Siclia, G., Reflexiones sobre el Sistema Educativo Español (pp. 283-312). Madrid: Centro de Estudios Ramón Areces. Recuperado de https://www.fundacionareces.es/recursos/doc/portal/2018/ 03/19/reflexiones-sistema-educativopdf.pdf

Tai, J., Ajjawi, R., Boud, D., Dawson, Ph. y Panadero, E. (2018). Developing evaluative judgement: enabling students to make decisions about the quality of work. In Higher Education, 76, 467-481. DOI: 10.1007/s10734-017-0220-3

Winstone N. (2019). Facilitating Students' Use of Feedback: Capturing and Tracking Impact Using Digital Tools. In Henderson M., Ajjawi R., Boud D., Molloy E. (Eds). The Impact of Feedback in Higher Education (pp. 207-223) Palgrave Macmillan, Cham. DOI: 10.1007/978-3-030-25112-3_132

Winstone, N. E., Nash, R. A., Rowntree, J. y Parker, M. (2017). 'It'd be useful, but I wouldn't use it': barriers to university students' feedback seeking and recipience. In Studies in Higher Education, 42(11), pp.

20262041. DOI: $10.1080 / 03075079.2015 .1130032$

Zimmerman, B. J. (2001). Theories of self-regulated learning and academic achievement: An overview and analysis. In Zimmerman, B. J. y D. H. Schunk (Eds). Self-regulated Learning and Academic Achievement: Theoretical Perspectives (pp. 1-37). London: Lawrence Erlbaum. 
120 ISSN0213-8646 | E-ISSN 2530-3791 • Revista Interuniversitaria de Formación delProfesorado, 95(34.2) (2020), 100-120 